\title{
Strong edge colorings of graphs and the covers of Kneser graphs
}

\author{
Borut Lužar, Edita Mačajováł Martin Škovierał Roman Sotákł \\ January 14, 2021
}

\begin{abstract}
A proper edge coloring of a graph is strong if it creates no bichromatic path of length three. It is well known that for a strong edge coloring of a $k$-regular graph at least $2 k-1$ colors are needed. We show that a $k$-regular graph admits a strong edge coloring with $2 k-1$ colors if and only if it covers the Kneser graph $K(2 k-1, k-1)$. In particular, a cubic graph is strongly 5-edge-colorable whenever it covers the Petersen graph. One of the implications of this result is that a conjecture about strong edge colorings of subcubic graphs proposed by Faudree et al. [Ars Combin. 29 B (1990), 205-211] is false.
\end{abstract}

Keywords: strong edge coloring, Petersen coloring, Kneser graph, odd graph, cubic graph, covering projection

\section{Introduction}

A strong edge coloring of a graph $G$ is a proper edge coloring with no bichromatic path of length three; in other words, each color class is an induced matching. The minimum number of colors for which $G$ admits a strong edge coloring is called the strong chromatic index, and is denoted by $\chi_{s}^{\prime}(G)$. In 1985, Erdős and Nešetřil proposed the following conjecture which was later published in [5] and updated by Faudree et al. [7] to fit the graphs with an even or odd maximum degree.

Conjecture 1.1 (Erdős, Nešetřil, 1988). The strong chromatic index of an arbitrary graph $G$ satisfies

$$
\chi_{s}^{\prime}(G) \leq\left\{\begin{array}{cl}
\frac{5}{4} \Delta(G)^{2}, & \text { if } \Delta(G) \text { is even } \\
\frac{1}{4}\left(5 \Delta(G)^{2}-2 \Delta(G)+1\right), & \text { if } \Delta(G) \text { is odd. }
\end{array}\right.
$$

*Faculty of Information Studies, Novo mesto, Slovenia. E-Mail: borut.luzar@gmail.com

$\dagger$ Comenius University, Bratislava, Slovakia. E-Mails: \{macajova, skoviera\}@dcs.fmph.uniba.sk

${ }^{\ddagger}$ Pavol Jozef Šafárik University, Košice, Slovakia. E-Mail: roman.sotak@upjs.sk 
Despite many efforts, this conjecture is still widely open and the best current upper bound $1.772 \Delta(G)^{2}$ (provided that $\Delta(G)$ is large enough) is due to Hurley et al. [11.

The motivation for this note comes from one of the two extant cases of the conjecture of Faudree et al. [7, Section 4] about strong edge colorings of subcubic graphs, that is, graphs with maximum degree 3 .

Conjecture 1.2 (Faudree, Schelp, Gyárfás, Tuza, 1990). Let $G$ be a graph with maximum degree 3. Then

(1) $\chi_{s}^{\prime}(G) \leq 10$

(2) $\chi_{s}^{\prime}(G) \leq 9$ if $G$ is bipartite,

(3) $\chi_{s}^{\prime}(G) \leq 9$ if $G$ is planar,

(4) $\chi_{s}^{\prime}(G) \leq 6$ if $G$ is bipartite and for every edge the sum of degrees of its endvertices is at most 5 ,

(5) $\chi_{s}^{\prime}(G) \leq 7$ if $G$ is bipartite with girth at least 6 ,

(6) $\chi_{s}^{\prime}(G) \leq 5$ if $G$ is bipartite with large girth.

The first four cases of Conjecture 1.2 have already been resolved. Case (1), which is just a special case of the conjecture of Erdős and Nešetřil, has been confirmed by Andersen [1] and by Horák et al. [10]. Case (2) was proved in 1993 by Steger and Yu [17, and Case (3), just recently, by Kostochka et al. [14. Case (4) was established by Wu and Lin [18]; it easily follows also from a result of Maydanskiy [15]. Up to our best knowledge, Cases (5) and (6) are still open, although several partial results confirming Case (6) are known [3, 4].

Our aim is to show that Case (6) of Conjecture 1.2 is false. In order to be able to produce infinitely many counterexamples, in Theorem 2.2 we characterize $k$-regular graphs with strong chromatic index $2 k-1$ as those which admit a covering projection onto the Kneser graph $K(2 k-1, k-1)$. In particular, when $k=3$, a cubic graph is strongly 5-edge-colorable if and only if it covers the Kneser graph $K(5,2)$. However, the latter is nothing but the Petersen graph. Subsequently, in Theorem 2.4, we construct bipartite cubic graphs of arbitrarily large girth that do not cover the Petersen graph. By the former result, their strong chromatic index must be at least 6 .

The last three sections of this paper are devoted to further aspects of strong $(2 k-1)$ colorings of $k$-regular graphs, with emphasis on the cubic case. In Section 3, we reflect on the fact, established in Section 2, that the Kneser graph $K(2 k-1, k-1)$ has a unique strong $(2 k-1)$-coloring up to automorphism. With the help of a result borrowed from the theory of voltage graphs we are able to provide an example of a cubic graph on 40 vertices which covers the Petersen graph and admits two substantially different strong 5-edge-colorings. In Section 4, we explain the relationship of strong 5-colorings of cubic graphs to the famous Petersen coloring conjecture, and in the final section we present several open problems. Among them, we propose a strengthening of Case (5) of Conjecture 1.2, its only remaining open case. 


\section{Main results}

Let $\phi$ be a proper edge coloring of a graph $G$. An edge $e$ of $G$ is said to be rich with respect to $\phi$ if all the edges adjacent to $e$ receive pairwise distinct colors. If $\phi$ is a strong edge coloring, then each edge must obviously be rich, and vice versa. In particular, every strong edge coloring of a $k$-regular graph requires at least $2 k-1$ colors. It has been shown in [7, Theorem 8] that this minimum is also sufficient if $G$ is the Kneser graph $K(2 k-1, k-1)$. Recall that the Kneser graph $K(m, n)$, with $m \geq 2 n+1$ and $n \geq 2$, is the graph whose vertices are the $n$-element subsets of a ground set of $m$ elements, say $\{1,2, \ldots, m\}$, and where two vertices are adjacent if and only if the two corresponding sets are disjoint. The Kneser graphs $K(2 k-1, k-1)$ are commonly known as the odd graphs $O_{k}$ and have been subject to numerous investigations (see for example the work of Biggs [2]). The smallest odd graph $K(5,2)$ is isomorphic to the Petersen graph.

Every odd graph $K(2 k-1, k-1)$ has a natural - or canonical - strong $(2 k-1)$ edge-coloring, which we denote by $\sigma_{k}$. It can be described as follows: for any edge $u v$ of $K(2 k-1, k-1)$ the set $u \cup v \subseteq\{1,2, \ldots, 2 k-1\}$ contains precisely $2 k-2$ elements, so we can set $\sigma_{k}(u v)$ to be the missing element of the ground set. It is easy to see that this coloring is indeed strong. The canonical strong 5-edge-coloring $\sigma_{3}$ of the Petersen graph is represented in Figure 1 .

Observe that every strong $(2 k-1)$-edge-coloring $\sigma$ of any $k$-regular graph $G$ induces a vertex coloring $\sigma^{\prime}$ of $G$ where every vertex $v$ is colored with the $(k-1)$-element set of colors that do not occur on the edges incident with $v$. We call $\sigma^{\prime}$ the derived vertex coloring. Since $\sigma$ is strong, the colors of any two adjacent vertices of $G$ are disjoint $(k-1)$-subsets; in particular, $\sigma^{\prime}$ is a proper vertex coloring. For the Petersen graph the derived coloring $\sigma_{3}^{\prime}$ is again indicated in Figure 1 .

It is quite remarkable that the edge coloring $\sigma$ can be uniquely reconstructed from the vertex coloring $\sigma^{\prime}$ : the edge $u v$ is colored with the element of the ground set not occurring in the set $\sigma^{\prime}(u) \cup \sigma^{\prime}(v)$. This fact readily implies that the canonical coloring is a unique strong $(2 k-1)$-edge-coloring of $K(2 k-1, k-1)$ up to automorphism of $K(2 k-1, k-1)$. Indeed, consider an arbitrary strong $(2 k-1)$-edge-coloring $\tau$ of $K(2 k-1, k-1)$. The derived vertex coloring $\tau^{\prime}$ associates with each vertex $v$ of $K(2 k-1, k-1)$ - which is a $(k-1)$-element subset of $\{1,2, \ldots, 2 k-1\}$ - another $(k-1)$-element subset $\tau^{\prime}(v)$ of the same set. In other words, $\tau^{\prime}$ sends a vertex of $K(2 k-1, k-1)$ to another such vertex. Since $\tau$ is strong, the assignment $v \mapsto \tau^{\prime}(v)$ is adjacency-preserving and injective on the neighbors of $v$. It means that $\tau^{\prime}$ determines a degree-preserving endomorphism $\alpha$ of $K(2 k-1, k-1)$, which necessarily must be an automorphism. The way how the canonical $(2 k-1)$-edge-coloring $\sigma_{k}$ was defined implies that $\alpha$ transforms $\sigma_{k}^{\prime}$ to $\tau^{\prime}$, and consequently $\sigma_{k}$ to $\tau$. Summing up, any two strong $(2 k-1)$-edge-colorings of $K(2 k-1, k-1)$ are equivalent under the action of its automorphism group. Taking into account the fact that every automorphism of $K(2 k-1, k-1)$ is induced by a permutation of the ground set [2, Statement 3.1] we can conclude that any two strong $(2 k-1)$-colorings of $K(2 k-1, k-1)$ can be obtained from each other by a permutation of colors.

We aim to prove that all $k$-regular graphs whose strong chromatic index equals $2 k-1$ are closely related to the Kneser graph $K(2 k-1, k-1)$, the relationship being a covering 


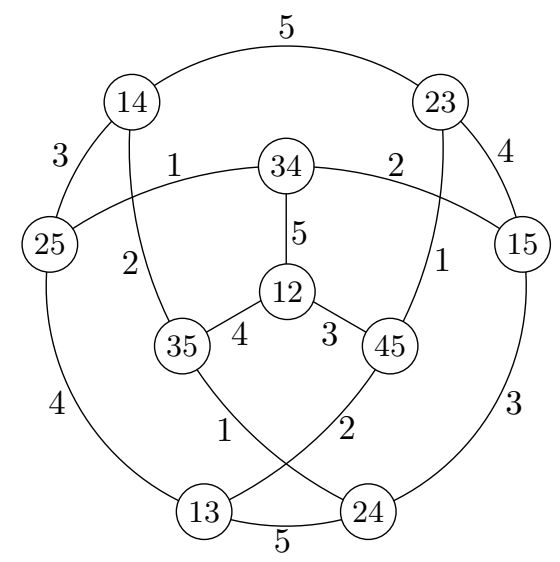

Figure 1: The unique strong 5-edge-coloring of the Petersen graph along with the derived proper vertex coloring

projection onto $K(2 k-1, k-1)$. The pertinent definitions are now in order.

A surjective graph homomorphism $f: \tilde{G} \rightarrow G$ is called a covering projection if for every vertex $\tilde{v}$ of $\tilde{G}$ the set of edges incident with $\tilde{v}$ is bijectively mapped onto the set of edges incident with $f(\tilde{v})$. (If $G$ is permitted to contain loops, then the definition has to be applied to the half-edges incident with $v$ rather than the edges themselves.) The graph $G$ is usually referred to as the base graph and $\tilde{G}$ as a covering graph or a lift of $G$. A graph $\tilde{G}$ covers $G$ if there exists such a covering projection.

It is well known (see [8], Section 2.3) that for every covering projection $f: \tilde{G} \rightarrow G$ there exists a positive integer $d$ such that every vertex of $G$ has exactly $d$ preimages and every edge of $G$ has exactly $d$ preimages; such a cover is said to be $d$-fold. For example, the Petersen graph admits a 5-fold covering projection onto the dumbbell graph (which consists of two adjacent vertices and a loop attached to each of them).

Covering graphs have been useful in numerous parts of graph theory, especially when a locally defined structure on the base graph can be 'lifted' to the covering graph. This is true, for example, for edge colorings, flows, embeddings on surfaces, and other graph structures. The following easy fact is in a similar vein.

Lemma 2.1. Let $f: \tilde{G} \rightarrow G$ be a covering projection of graphs. If $G$ is strongly n-edgecolorable for some integer $n$, then so is $\tilde{G}$.

Proof. Let $\phi$ be a strong $n$-edge-coloring of $G$. Define an edge coloring $\tilde{\phi}$ of $\tilde{G}$ by setting $\tilde{\phi}(x)=\phi(f(x))$ for each edge $x$ of $\tilde{G}$. As previously mentioned, every edge of $G$ with respect to $\phi$ is rich. The definition of a covering immediately implies that the same holds for each edge of $\tilde{G}$ with respect to $\tilde{\phi}$. Therefore $\tilde{\phi}$ is a strong $n$-edge-coloring of $\tilde{G}$.

Now we are ready for our main results.

Theorem 2.2. The strong chromatic index of a $k$-regular graph $G$ equals $2 k-1$ if and only if $G$ covers the Kneser graph $K(2 k-1, k-1)$. 
Proof. The backward implication is a direct consequence of Lemma 2.1, so we are left with the forward implication.

Let $\sigma$ be a strong edge coloring of a $k$-regular graph $G$ with $2 k-1$ colors from the set $\{1,2, \ldots, 2 k-1\}$. Without loss of generality we may assume that $G$ is connected. To define a covering projection $f: G \rightarrow K(2 k-1, k-1)$ we use the derived vertex coloring $\sigma^{\prime}$ of $G$. Recall that for each vertex $v$ of $G$ the color $\sigma^{\prime}(v)$ is a $(k-1)$-subset of $\{1,2, \ldots, 2 k-1\}$. Thus there is a unique vertex $\bar{v}$ of $K(2 k-1, k-1)$ such that $\sigma^{\prime}(v)=\bar{v}$. Define $f$ by sending $v$ to $\bar{v}$. The mapping is clearly correctly defined.

We first observe that $f: G \rightarrow K(2 k-1, k-1)$ is a homomorphism. To see this, note that the colors of adjacent vertices in $G$ are disjoint $(k-1)$-elements sets. It follows that $f$ sends adjacent vertices $u$ and $v$ to disjoint sets $\bar{u}$ and $\bar{v}$. However, in $K(2 k-1, k-1)$ such vertices are adjacent. Therefore $f$ sends adjacent vertices to adjacent vertices.

To show that $f$ is a covering projection we need to check that $f$ takes the neighborhood of every vertex bijectively to $K(2 k-1, k-1)$, and that $f$ is surjective. Consider an arbitrary vertex $v$ of $G$, and note that the $k$ neighbors $u_{i}$ of $v$, where $1 \leq i \leq k$, receive from $\sigma^{\prime}$ pairwise distinct colors $\sigma^{\prime}\left(u_{i}\right)$. Since $f$ sends each $u_{i}$ to the vertex $\bar{u}_{i}=\sigma^{\prime}\left(u_{i}\right)$ in $K(2 k-1, k-1)$, it takes the $k$ neighbors of $v$ to $k$ distinct neighbors of $f(v)$, as required.

Finally, to check that $f$ is surjective it is sufficient to realize that $f(G)$ is a connected subgraph of $K(2 k-1, k-1)$ and that $f(G)$ is $k$-regular. Therefore $f(G)=K(2 k-1, k-1)$, which proves that $f$ is a covering projection.

For cubic graph the previous theorem amounts to the following.

Corollary 2.3. The strong chromatic index of a cubic graph $G$ equals 5 if and only if $G$ covers the Petersen graph.

Our next theorem refutes Case (6) of Conjecture 1.2 .

Theorem 2.4. There exist bipartite cubic graphs with arbitrarily large girth and strong chromatic index at least 6.

Proof. We construct an infinite sequence $\left(G_{n}\right)_{n \geq 1}$ of connected bipartite cubic graphs such that $G_{n}$ has girth $2^{n}$ and order a power of 2 . Since the order of any graph that covers the Petersen graph is a multiple of 10 , it follows that $G_{n}$ does not cover the Petersen graph for any $n \geq 1$. From Corollary 2.3 we get that $\chi_{s}^{\prime}\left(G_{n}\right) \geq 6$ for each $n \geq 2$.

We now construct the sequence by induction on $n$. For the starting graph $G_{1}$ we take the cubic graph consisting of two vertices and three parallel edges, which is connected, bipartite, and has girth 2. Assume that we have already constructed the graph $G_{n}$ for some $n \geq 1$. By the induction hypothesis, $G_{n}$ is connected and bipartite with girth $2^{n}$ and order a power of 2 . To create $G_{n+1}$, we employ the construction of Exoo and Jajcay described in the proof of Theorem 3.1 of [6]. Their method uses a $\mathbb{Z}_{2}$-homology voltage assignment on a connected graph $H$ of girth $g$ to produce a $2^{\beta(H)}$-fold covering projection $\tilde{H} \rightarrow H$ with $\tilde{H}$ connected of girth $2 g$, where $\beta(G)$ denotes the cycle rank (Betti number) of $G$. (We refer the reader for details to [6].) If we apply this construction to $G_{n}$, we obtain a connected graph $G_{n+1}$ of girth $2^{n+1}$ and order $2^{\beta\left(G_{n}\right)} m_{n}$, where $m_{n}$ is the order of $G_{n}$. Since $m_{n}$ is a power of 2 , so is the order of $G_{n+1}$. Furthermore, $G_{n+1}$ is bipartite 
because any covering graph over a bipartite base graph is bipartite. This concludes the construction of $\left(G_{n}\right)_{n \geq 1}$ as well as the entire proof.

Remark 2.5. The reader can check that the graph $G_{2}$ constructed in the previous proof is isomorphic to the graph of the 3 -cube $Q_{3}$, whose strong chromatic index equals 6 . Since the composition of covering projections is again a covering projection, each $G_{n}$ with $n \geq 3$ covers $G_{2}$, and therefore it is strongly 6 -edge-colorable by Lemma 2.1 . Theorem 2.2 now implies that $\chi_{s}^{\prime}\left(G_{n}\right)=6$ for each $n \geq 2$.

\section{Equivalence of coverings and colorings}

We have proved that the odd graph $K(2 k-1, k-1)$ admits a unique strong $(2 k-1)$ edge-coloring up to automorphism. It is therefore natural to ask whether the same holds for the graphs that cover it. As we shall see in this section, the answer is negative, which at the first glance might seem to be rather counter-intuitive.

We first show that the problem of finding two essentially different strong $(2 k-1)$ edge-colorings of a $k$-regular graph is closely related to the problem of finding two nonequivalent covering projections of the same $k$-regular graph onto the odd graph $K(2 k-1$, $k-1)$.

We call two edge colorings $\phi_{1}$ and $\phi_{2}$ of a graph $G$ equivalent if there exists an automorphism $\alpha$ of $G$ such that $\phi_{2}=\phi_{1} \alpha$. Similarly, we say that two covering projections $f_{1}: G_{1} \rightarrow G$ and $f_{2}: G_{2} \rightarrow G$ are equivalent if there exists an isomorphism $\xi: G_{2} \rightarrow G_{1}$ such that $f_{2}=f_{1} \alpha$.

The following theorem shows that for strong $(2 k-1)$-edge-colorings of $k$-regular graphs equivalence of colorings coincides with equivalence of coverings.

Theorem 3.1. Every strong $(2 k-1)$-edge-coloring $\sigma$ of a $k$-regular graph $G$ determines a unique covering projection $f_{\sigma}: G \rightarrow K(2 k-1, k-1)$. Moreover, two such colorings $\sigma$ and $\tau$ are equivalent if and only if the corresponding covering projections $f_{\sigma}$ and $f_{\tau}$ of $G$ are equivalent.

Proof. Recall that given a strong $(2 k-1)$-edge-coloring $\sigma$ of an arbitrary graph $G$ we have defined a covering projection $f$ by sending an arbitrary vertex $v$ of $G$ to the vertex $\sigma^{\prime}(v)$, where $\sigma^{\prime}$ is the derived vertex coloring of $G$ with colors being the $(k-1)$-element subsets of the $(2 k-1)$-element set. This is the required covering projection $f_{\sigma}$ corresponding to the coloring $\sigma$.

Assume that $\sigma$ and $\tau$ are equivalent strong $(2 k-1)$-edge-colorings of $G$, and let $\alpha$ be an automorphism of $G$ such that $\tau=\sigma \alpha$. It follows that $\tau^{\prime}=\sigma^{\prime} \alpha$ and therefore, by the definition of the covering projection corresponding to a strong $(2 k-1)$-edgecoloring, $f_{\tau}=f_{\sigma} \alpha$. Conversely, if covering projections $f_{\sigma}, f_{\tau}: G \rightarrow K(2 k-1, k-1)$ are equivalent, then there exists an automorphism $\beta$ of $G$ such that $f_{\tau}=f_{\sigma} \beta$. The latter can be rewritten as $\tau^{\prime}=\sigma^{\prime} \beta$. However, the way how the original strong $(2 k-1)$-edge-coloring can be reconstructed from the derived vertex coloring implies that $\tau=\sigma \beta$, which means that the colorings $\sigma$ and $\tau$ are equivalent. 
What remains to be done is to find a $k$-regular graph $G$ that admits two non-equivalent covering projections on the Kneser graph $K(2 k-1, k-1)$. We do it for $k=3$, in which case $K(2 k-1, k-1)$ coincides with the Petersen graph. For this purpose we need to recall several notions pertaining to the theory of graph covers.

First of all, it will be convenient to regard each edge (including the loops) as a pair of oppositely oriented darts. Each dart $x$ directed from $u$ to $v$ has its unique inverse $x^{-1}$ directed from $v$ to $u$. The set of all darts of a graph $K$ is denoted by $D(K)$. The symmetric group of all permutations of the $d$-element set $\{1,2, \ldots, d\}$ is denoted by $S_{d}$; it acts on $\{1,2, \ldots, d\}$ on the right.

A permutation voltage assignment on a graph $K$ is a mapping $\kappa: D(K) \rightarrow S_{d}$ such that $\kappa\left(x^{-1}\right)=\kappa(x)^{-1}$ for each dart $x \in D(K)$. For convenience, we often denote $\kappa(x)$ by $\kappa_{x}$. Every permutation voltage assignment $\kappa$ on $K$ gives rise to the derived graph $K^{\kappa}$ for $K$, or the lift of $K$, which is defined as follows. Set $V\left(K^{\kappa}\right)=V(K) \times\{1,2, \ldots, d\}$, $D\left(K^{\kappa}\right)=D(K) \times\{1,2, \ldots, d\}$, and for each dart $x=u v$ and $i \in\{1,2, \ldots, d\}$ let the lifted dart $(x, i)$ join $(u, i)$ to $\left(v,(i) \kappa_{x}\right)$. It is easy to see that the natural projection $p_{\kappa}: K^{\kappa} \rightarrow K$ which erases the second coordinate is a covering projection. Moreover, a classical result of the theory of voltage graphs states that every $d$-fold covering projection $\tilde{K} \rightarrow K$ is equivalent to the natural projection $K^{\kappa} \rightarrow K$ for a suitable permutation voltage assignment $\kappa$ on $K$ with values in $S_{d}$, see [8, Theorem 2.4.5].

Let us henceforth assume that the base graph $K$ is connected. Pick a spanning tree $T$ of $K$ and let $r$ be an arbitrary vertex of $K$, the root. For any vertex $w$ of $K$ let $T(w)$ denote the unique directed path from the root to $w$, encoded as a sequence of darts, and let $T(w)^{-1}$ be the inverse path. Further, for any dart $z=u v$ whose underlying edge is not contained in $T$ define the permutation $\theta(z) \in S_{d}$ by taking the product of voltages (that is, values of $\kappa$ ) on the closed walk $T(u) z T(v)^{-1}$, rooted at $r$, in the order determined by the walk and starting from the root. If we set $\kappa^{\prime}(x)=\theta(x)$ whenever $x$ is a cotree dart and $\kappa^{\prime}(x)=$ id otherwise, we obtain a new voltage assignment $\kappa^{\prime}$ on $K$ called the $(T, r)$-reduction of $\kappa$.

The concept of a $(T, r)$-reduction of a voltage assignment is quite useful. For example, it can be shown that the derived graph $K^{\kappa}$ is connected if and only if the voltages of $\kappa^{\prime}$ generate a transitive subgroup of $S_{d}$. What is more important for us, it can be used to determine whether or not two covering projections are equivalent. The respective result is taken from [16, Theorem 2].

Theorem 3.2. Let $\kappa$ and $\lambda$ be permutation voltage assignments on a connected graph $K$, both having their values in the symmetric group $S_{d}$, and let $\kappa^{\prime}$ and $\lambda^{\prime}$ be their $(T, r)$ reductions. The natural projections $p_{\kappa}$ and $p_{\lambda}$ are equivalent if and only if there exists an inner automorphism $\gamma$ of $S_{d}$ such that $\lambda^{\prime}=\gamma \kappa^{\prime}$.

Now we are prepared to describe an example of a cubic graph that covers the Petersen graph and has two non-equivalent strong 5-edge-colorings. It is depicted in Figure 3.

Example 3.3. Consider the permutation voltage assignments $\kappa$ and $\lambda$ on the Petersen graph with values in $S_{4}$ which are represented in Figure 2; the edges not labelled carry the trivial voltage id (in both directions). The values attached to all the edges are involutions, 
$\kappa$

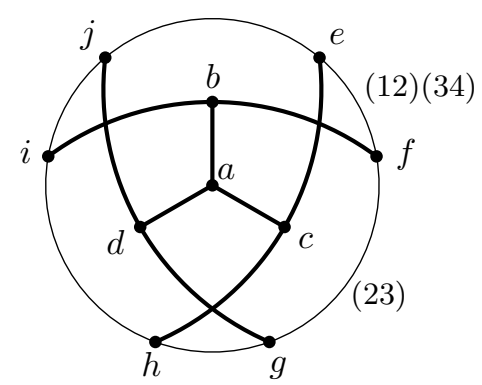

$\lambda$

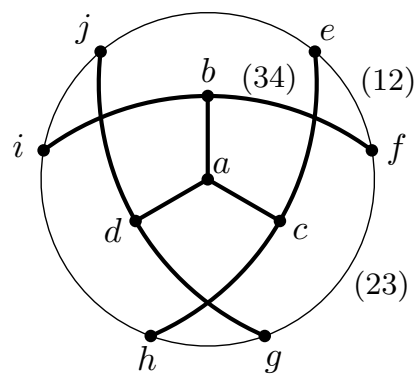

Figure 2: Permutation voltage assignments $\kappa$ and $\lambda$ on the Petersen graph with values in $S_{4}$.

which means that they unambiguously represent the respective voltage assignments. The corresponding lifts are isomorphic graphs as can be easily detected from Figure 3 for simplicity, a vertex $(v, i)$ is denoted by $v_{i}$. We claim that the natural projections $p_{\kappa}: P^{\kappa} \rightarrow P$

$\kappa$

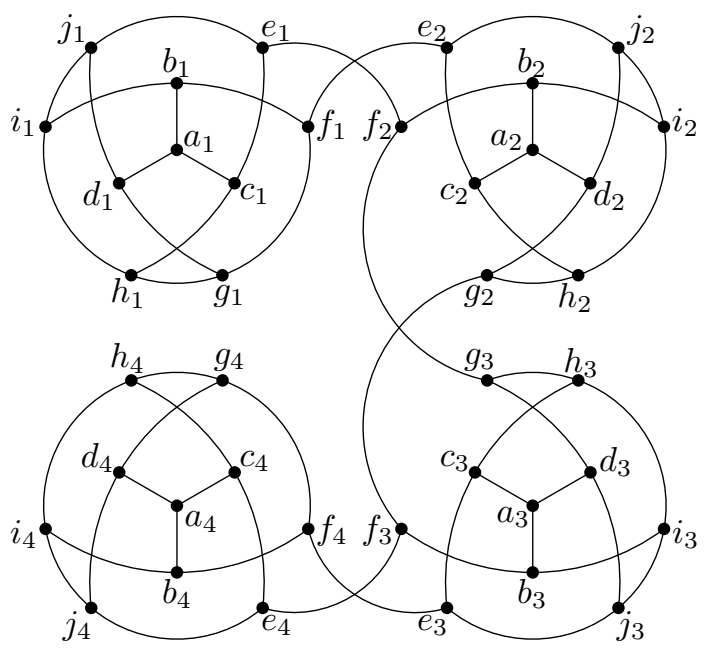

$\lambda$

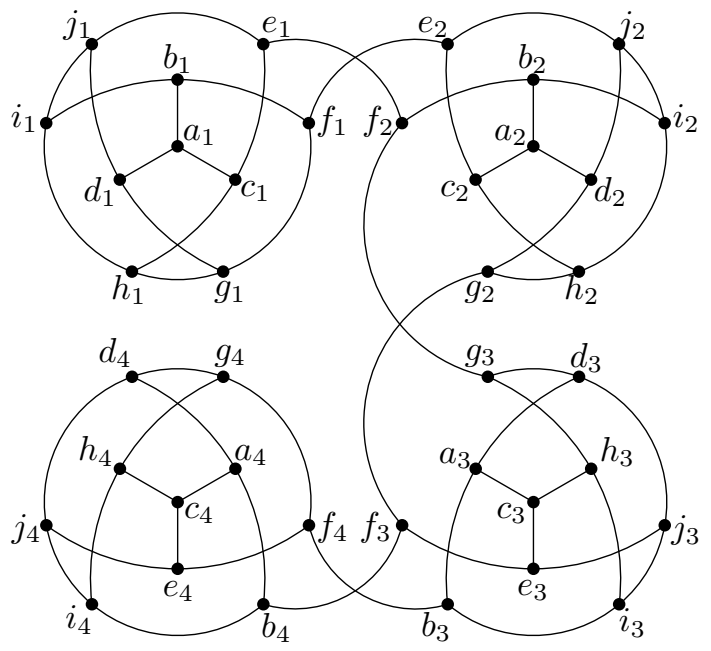

Figure 3: The lifts of the Petersen graph corresponding to the permutation voltage assignments $\kappa$ and $\lambda$.

and $p_{\lambda}: P^{\lambda} \rightarrow P$, where $P$ denotes the Petersen graph, are not equivalent. To see it, pick the spanning tree $T$ indicated in Figure 2 by bold lines and choose the central vertex $a$ to be the root. Clearly, the $(T, a)$-reduction $\kappa^{\prime}$ of $\kappa$ coincides with $\kappa$. The $(T, a)$-reduction $\lambda^{\prime}$ of $\lambda$ is shown in Figure 4, the value $\lambda^{\prime}(g f)$ is not an involution and holds for the indicated direction. By comparing $\kappa^{\prime}$ and $\lambda^{\prime}$ we immediately conclude that no inner automorphism $\gamma$ of $S_{d}$ such that $\lambda^{\prime}=\gamma \kappa^{\prime}$ can exist, because every inner automorphism preserves the cycle structure of permutations. Theorem 3.2 now implies that the covering projections 


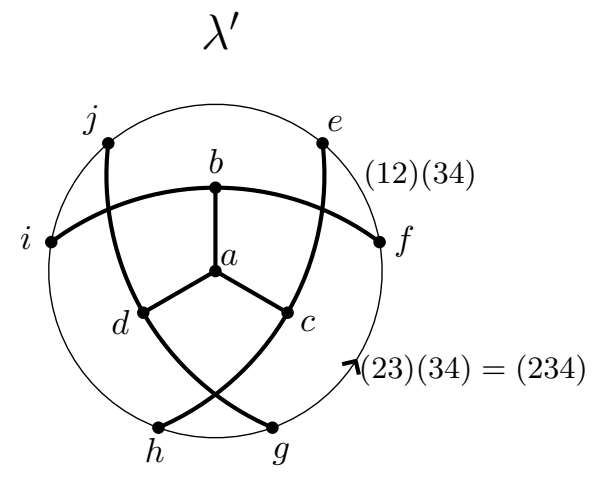

Figure 4: The $(T, a)$-reduction $\lambda^{\prime}$ of $\lambda$.

$p_{\kappa}$ and $p_{\lambda}$ are not equivalent. Further, from Theorem 3.1 we conclude that there exist strong 5-edge-colorings $\sigma$ and $\tau$ of the graph shown in Figure 3 such that $f_{\sigma}$ is equivalent to $p_{\kappa}$ and $f_{\tau}$ is equivalent to $p_{\lambda}$. They can be constructed simply by lifting the strong 5 -edge-coloring $\sigma_{3}$ of the Petersen graph via $p_{\kappa}$ and $p_{\lambda}$, respectively. By the same theorem, the colorings $\sigma$ and $\tau$ are not equivalent.

\section{Strong, normal, and Petersen colorings}

Corollary 2.3 links strong edge colorings of cubic graphs to two other interesting types of edge colorings of cubic graphs - normal colorings and Petersen colorings - and through them to the outstanding Petersen coloring conjecture of Jaeger [13].

Conjecture 4.1 (Jaeger, 1988). Every bridgeless cubic graph admits a Petersen coloring.

For a cubic graph $G$ a mapping $\xi: E(G) \rightarrow E(P)$ is said to be a Petersen coloring if any two adjacent edges of $G$ are mapped to adjacent edges of the Petersen graph. As a consequence, for every vertex $v$ of $G$ the three edges incident with $v$ are mapped to three edges incident with a vertex of $P$ (as $P$ is triangle-free); in particular, $\xi$ is a proper edge coloring. Nevertheless, a Petersen coloring need not be a homomorphism $G \rightarrow P$ because the induced mapping between the vertex sets need not send adjacent vertices of $G$ to adjacent vertices of $P$ (for example, it can send them to the same vertex). If $\xi$ does map adjacent vertices to adjacent ones, then it determines a covering projection $G \rightarrow P$. Conversely, every covering projection $G \rightarrow P$ gives rise to a Petersen coloring of $G$.

In [12, Section 5] Jaeger proved that a cubic graph admits a Petersen coloring if and only if it has a 5-edge-coloring which he termed 'normal'. A proper 5-edge-coloring $\phi$ of a cubic graph $G$ is said to be normal if for every edge $e$ of $G$ the number of colors on the edges adjacent to $e$ is either 2 or 4 , but never 3. In the former case, $e$ is called poor, as opposed to rich (introduced in Section 2), which corresponds to the latter case. Clearly, a normal 5-edge-coloring with no poor edges is strong.

By combining these observations with Corollary 2.3 we obtain the following four equivalent statements. 
Theorem 4.2. Let $G$ be cubic graph. Then the following statements are equivalent:

(i) $\chi_{s}^{\prime}(G)=5$;

(ii) $G$ covers the Petersen graph;

(iii) $G$ admits a normal 5-edge-coloring in which every edge is rich;

(iv) G admits a Petersen coloring which is a homomorphism.

\section{Conclusion}

Coloring subcubic graphs with five colors without creating a bichromatic path of length 3 is very restrictive. For example, we already need five colors when there are two adjacent vertices of degree 3 in a graph. Therefore, the existence of infinitely many cubic graphs admitting a strong 5-edge-coloring is quite surprising.
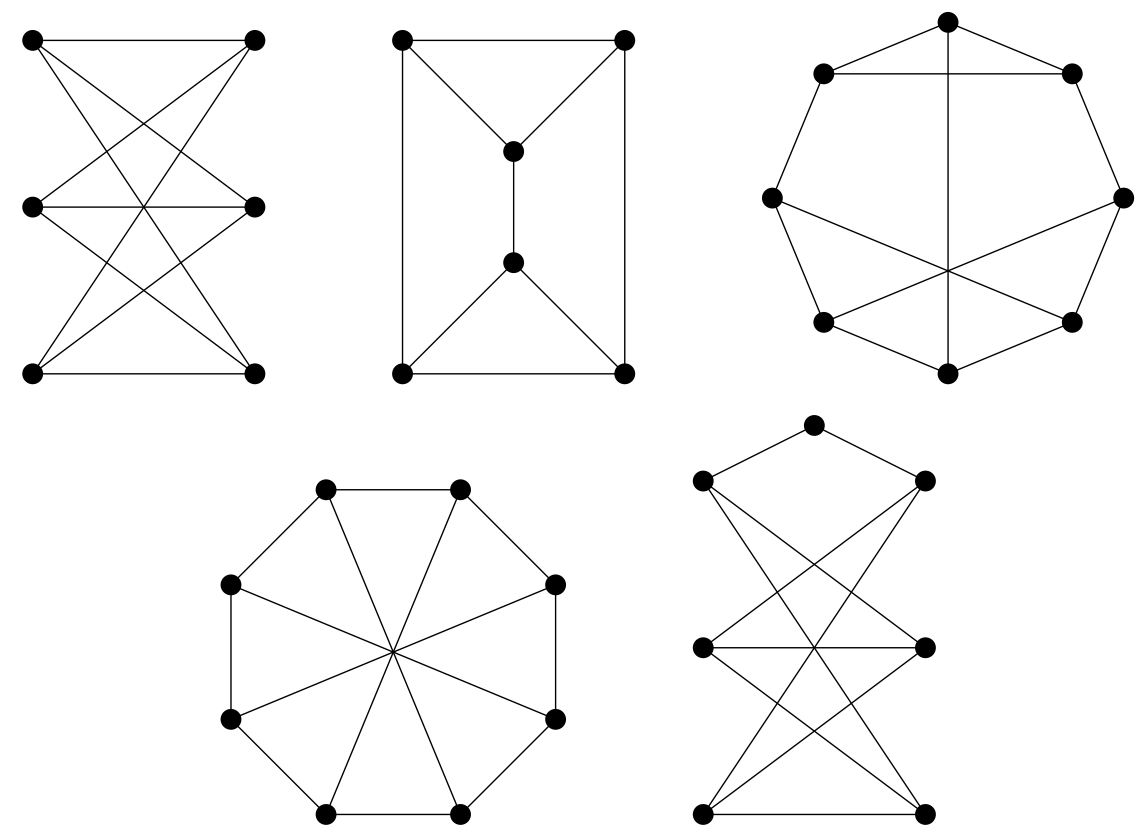

Figure 5: Subcubic graphs with high strong chromatic indices; the three graphs above need nine colors and the two graphs below ten colors for any strong edge coloring.

What if we have more available colors? It is already known that the Heawood graph, a cubic graph on 14 vertices of girth 6 , has strong chromatic index 7 . Computational results reveal that there are two and six cubic graphs of girth at least 6 on 16 and 18 vertices, respectively, for which six colors are not sufficient. It is even not enough to slightly increase the girth. The Tutte 8-cage, a cubic graph of girth 8 on 30 vertices, does not admit any strong 6-edge-coloring, nor do five cubic graphs of girth 8 on 38 vertices. This encourages us to ask the following. 
Question 5.1. Is there a constant $C$ such that every cubic graph of girth at least $C$ admits a strong 6-edge-coloring?

Perhaps one can find a characterization of cubic graphs admitting a strong 6-edgecoloring similar to the one presented in this paper for five colors. However, it is likely that the condition of being bipartite will not play any role in answering the above question, because Tutte's 8-cage is bipartite.

Having seven colors available, it seems that cubic graphs become strongly colorable as soon as they do not contain short cycles. Our computational experiments show that all cubic graphs of girth at least 5 on at most 26 vertices and all bridgeless subcubic graphs of girth at least 5 on at most 18 vertices admit a strong 7-edge-coloring. We therefore propose the following conjecture, which strengthens Case (5) of Conjecture 1.2 .

Conjecture 5.2. Let $G$ be a subcubic graph of girth at least 5. Then

$$
\chi_{s}^{\prime}(G) \leq 7 \text {. }
$$

We remark that without restricting girth eight colors are still not sufficient to color all bridgeless subcubic graphs (see Figure 5 for some examples of graphs that need more colors). However, as suggested in [9] on the basis of computational evidence, it seems that nine colors should suffice to color all bridgeless subcubic graphs that are not isomorphic to either $K_{3,3}$ with one subdivided edge or to the 8-vertex Möbius ladder known as the Wagner graph.

Conjecture 5.3 (Hocquard, Lajou, Lužar, 2020). Every bridgeless subcubic graph not isomorphic to the Wagner graph and the complete bipartite graph $K_{3,3}$ with one edge subdivided admits a strong 9-edge-coloring.

In fact, exhaustive computational search indicates that there are no bridgeless subcubic graphs on more than 12 vertices and strong chromatic index at least 9 (apart from the graphs in Figure 5 and four graphs on 12 vertices). Thus we can strengthen the above conjecture to the following.

Conjecture 5.4. If $G$ is a bridgeless subcubic graph on at least 13 vertices, then

$$
\chi_{s}^{\prime}(G) \leq 8
$$

Acknowledgment. The first author was partially supported by the Slovenian Research Agency Program P1-0383 and the project J1-1692. The second and the third author received partial support from APVV-19-0308 and VEGA 1/0813/18. The fourth author was supported by APVV-19-0153.

\section{References}

[1] L. D. Andersen, The strong chromatic index of a cubic graph is at most 10, Discrete Math. 108 (1992), 231-252. 
[2] N. Biggs, Some odd graph theory, Second International Conference on Combinatorial Mathematics, Annals of the New York Acad. Sci. 319 (1979), 71-81.

[3] O. V. Borodin, A. O. Ivanova, Precise upper bound for the strong edge chromatic number of sparse planar graphs, Discuss. Math. Graph Theory 33 (2013), 759-770.

[4] P. DeOrsey, M. Ferrara, N. Graber, S. G. Hartke, L. L. Nelsen, E. Sullivan, S. Jahanbekam, B. Lidický, D. Stolee, J. White, On the strong chromatic index of sparse graphs, Electron. J. Combin. 25 (2018), \#P3.18.

[5] P. Erdős, Problems and results in combinatorial analysis and graph theory, Discrete Math. 72 (1988), 81-92.

[6] G. Exoo, R. Jajcay, On the girth of voltage graph lifts, Europ. J. Combin. 32 (2011), $554-562$.

[7] R. J. Faudree, R. H. Schelp, A. Gyárfás, Z. Tuza, The strong chromatic index of graphs, Ars Combin. 29B (1990), 205-211.

[8] J. L. Gross, T. W. Tucker, Topological Graph Theory, Wiley, New York, 1987.

[9] H. Hocquard, D. Lajou, B. Lužar, Between proper and strong edge-colorings of subcubic graphs, in: Gasieniec L., Klasing R., Radzik T. (eds.), Combinatorial Algorithms, IWOCA 2020, Lecture Notes in Comput. Sci. vol. 12126, Springer, pp. 355-367.

[10] P. Horák, H. Qing, W. T. Trotter, Induced matchings in cubic graphs, J. Graph Theory 17 (1993), 151-160.

[11] E. Hurley, R. de Joannis de Verclos, R. J. Kang, An improved procedure for colouring graphs of bounded local density ArXiv Preprint, 2020, https://arxiv.org/abs/2007.07874.

[12] F. Jaeger, On five-edge-colorings of cubic graphs and nowhere-zero flow problems, Ars Combin. 20B (1985), 229-244.

[13] F. Jaeger, Nowhere-zero flow problems, in: L. W. Beineke, R. J. Wilson (Eds.), Selected Topics in Graph Theory Vol. 3, Academic Press, London, 1988, pp. 71-95.

[14] A. V. Kostochka, X. Li, W. Ruksasakchai, M. Santana, T. Wang, G. Yu, Strong chromatic index of subcubic planar multigraphs, Europ. J. Combin. 51 (2016), 380397.

[15] M. Maydanskiy, The incidence coloring conjecture for graphs of maximum degree 3. Discrete Math. 292 (2005), 131-141.

[16] M. Škoviera, A contribution to the theory of voltage graphs, Discrete Math. 61 (1986), 281-292.

[17] A. Steger, M.-L. Yu, On induced matchings, Discrete Math. 120 (1993), 291-295.

[18] J. Wu, W. Lin, The strong chromatic index of a class of graphs, Discrete Math. 308 (2008), 6254-6261. 